\title{
Why Asymptomatic COVID19 Patient Could Face a Sudden Death
}

\author{
Amr Zaher ${ }^{1}$ and Sara Ali ${ }^{* 2}$ \\ ${ }^{1}$ National Heart Institute, Egypt \\ ${ }^{2}$ Zoology Department, Ain Shams University, Egypt \\ *Corresponding author: Sara Ali, MSc Degree in Physiology, Department of Zoology, College of Science, Ain Shams \\ University, Cairo-Egypt; sara.m.refaat@gmail.com
}

Received 07 February 2021;

Accepted 05 March 2021;

Published 18 March 2021

\begin{abstract}
Since the novel SARS-COV 2 has emerged from its inception in December 2019 till November 2020 about 68,587,138 infected cases,47,482,639 recovered, and 1,563,468 deaths ${ }^{[1,2]}$. Most of the death cases were diagnosed with critically ill pneumonia correlated with acute respiratory distress syndrome (ARDS) ${ }^{[2]}$. All of the previous numbers mostly have been recorded based on the patients who displayed symptoms whether from the most common symptoms like fatigue, cough, fever or severe symptoms as trouble breathing, persistent pain, or pressure in the chest. However, there are many infected people but didn't show any symptoms although they were developing COVID 19 complications, and some of them even died before know that they had a COVID 19. This is one of the most mysterious actions of coronavirus and bewildered the physicians how the patient could lose his oxygen gradually without panting to catch up his breath. In other words, asymptomatic patients who look completely fine don't necessarily far from the risk; they perhaps internally suffer from silent hypoxia or lung damage.
\end{abstract}

Keywords: COVID19, asymptomatic patient, silent Hypoxia, silent lung damage, and ground-glass opacities.

\section{Background}

In early December 2019, Chinese authorities identified a novel coronavirus, SARS-CoV-2 known as Coronavirus Disease 2019 (COVID-19). This virus has been launched in Wuhan, Hubei Province, China. The virus can cause a wide span of symptoms starting from mild upper respiratory tract infection to severe pneumonia and even death. Because of its rapid spread worldwide on March 11, 2020, the World Health Organization declared COVID-19 is a pandemic.

\section{Introduction}

Coronavirus ramifications are renowned for two faces; one of them appears in (fatigue, cough, fever, and taste loss and smell in some cases). While the second face could appear in a severe acute respiratory syndrome leaving behind a patient in intensive care, panting for breath, in imminent need of artificial ventilation ${ }^{[3,4]}$. However, recently, there is a third face for Coronavirus has appeared can be described as (Thief) as it steals the person's life without any alert or symptoms. This face is one of the most conundra the physicians try to find an interpretation. Therefore, if someone has confirmed a positive coronavirus without symptoms, shouldn't only stay at home, but needs to follow-up as he is at risk of developing COVID 19 complications.

In March, on the Diamond Princess cruise ship, out of 3,700 passengers on the board, 712 patients confirmed positive coronavirus. Not all the positive patients had symptoms, but 331 of them had no symptoms at all. The story didn't end in this chapter, for further investigations; the researchers took a random sample of 67 asymptomatic patients for examination by (CT) scans. The surprise when the researchers found half of this sample had lung abnormalities of ground-glass opacities (GGO) ${ }^{[5]}$.

Further, in a recent study at Wuhan University, 58 asymptomatic patients diagnosed with fluorescence reversetranscriptase-polymerase chain reaction (FRT-PCR) assays and high-resolution $\mathrm{CT}$; showed evidence of pneumonia and the predominant feature of CT images was GGO ${ }^{[6]}$. Therefore, the researchers consider the COVID 19 asymptomatic patients don't out of the dangerous, as although they might look totally in good health, they might be internally suffering from silent lung damage.

Continuing to the inscrutable behaviors of Coronavirus, some of the asymptomatic patients suffer from lack of oxygen which silently takes their breath away known for (silent hypoxia), and when they realize that they in danger might be too late. On April 20, in the New York Times, Dr Levitan is an emergency doctor has published his observations during ten days of treating 
COVID pneumonia at Bellevue Hospital. Dr Levitan said when he examined patients who passed out for no obvious reasons by CT scan, he coincidentally found COVID pneumonia. And although their oxygen was significantly low; they didn't show or complain about any sensation of shortness of breath or discomfort ${ }^{[7]}$.

Similarly, in Maharashtra, India, after the plethora of death cases that resulted from COVID19, the public health department has realized that the main murderer for most of the death cases was silent hypoxia. According to Dr. Archana Patil, director of State Health Services, most of those death cases were asymptomatic patients who reached the hospital too late. She added that those patients were infected by COVID19 four to seven days before without complaining about any discomfort to warn them until they came to the hospital with a critical case of breath problems ${ }^{[8]}$.

The above case reports have raised questions and doubts around the insidious face of COVID19, which threatens life without warning. In the following discussion, we try to expose some reasons that could be the key for such COVID19 ambiguous cases. Also, based on the researchers' and physicians' recommendations, we provide some advice to asymptomatic COVID 19 patients to steer clear of prospective silent lung damage or silent hypoxia.

\section{Aim of the review}

In this review, we try to find the possible answers to the most common baffled situations around COVID19 at the moment. This review contains accurate data and information at the publication time. However, this information could be changed regarding the last updating surrounding the COVID19 situation. Therefore, the reader always should keep following the updated news and recommendations released by the WHO and Centers for Disease Control CDC.

\section{Method}

Using the keywords of COVID19, asymptomatic patients, silent lung damage, silent hypoxia, and ground-glass opacities (GGO) for the search in all literature and case reports related to the review's question which have been published during (2020) in PubMed medical database, World Health Organization database, or other medical international journals by using google.

\section{Discussion}

Researchers have reported from 16 different groups (prison inmates, cruise ship passengers and nursing home residents) that COVID19 asymptomatic patients as many as $45 \%$ of the whole COVID 19 patients. Moreover, they have recorded more than half of asymptomatic patients had lung signs of lung damage ${ }^{[9]}$. Therefore, throughout the last few months, there have been several discussions to articulate to what extent the asymptomatic patients can silently develop COVID19 complications and how they could be at risk of sudden death ${ }^{[10]}$.

The researchers reported in the primary analysis among 858 non-duplicate studies, seven studies include 231 cases with no symptoms $63 \%$ of them confirmed lung abnormalities in CT particularly ground ground-glass opacities (GGO) ${ }^{[11]}$. Ground ground-glass opacities (GGOs) is the pattern that can be found in CT scans when there is an illness in the lung. The radiologists can identify it by its grey patches while the normal lung tissue appears black ${ }^{[12]}$. In the CT image, the healthy lung appears relatively dark because the air in the air sacs has a low density comparing to lung tissue. But, when any substance rather than air fills the air sacs, its density increases; therefore, the color appears on CT lighter or grey patches (Figure 1) ${ }^{[13]}$.

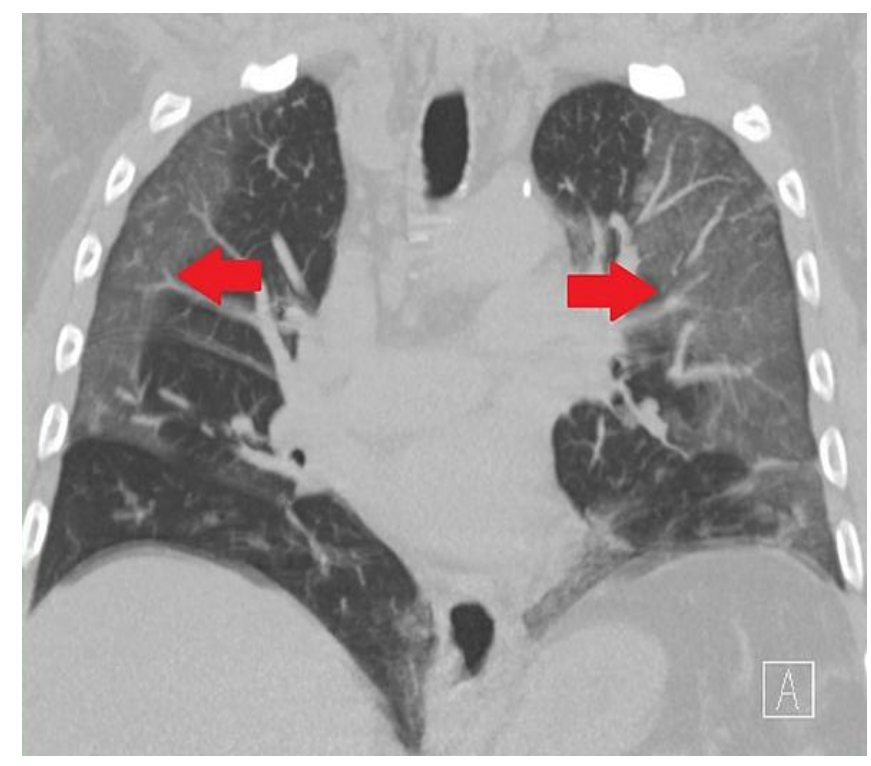

Figure 1: CT image of COVID19 patient, showing the difference between the normal lung tissue (the dark area) to ground-glass opacities (grey area) (Quoted from wikipedia.org)

GGO doesn't only correlate to COVID 19; but it is also related to several other diseases such as infections, interstitial lung disease, pulmonary edema, and lung cancers ${ }^{[12,14,15]}$. However, one of the most common illness correlated to GGOs is viral pneumonia. Thus, GGO in a CT scan is evidence of COVID-19-related pneumonia or lung inflammation of viral infection ${ }^{[12]}$.

Recently, the researchers took a perusal look at the clinical features of GGO in the 121 patients at four centers. Although GGO exists in different diseases; however, what the researchers saw in the COVID19 patients was quite different. COVID GGO has a round shape which is not the usual shape in the other lung abnormalities. Besides, GGO of COVID 19 has a distinguished pattern as it distributes in specific areas in the lung, such as lower lobes and periphery; and it can be seen multifocally and bilaterally as well (Figure 2) ${ }^{[16]}$.

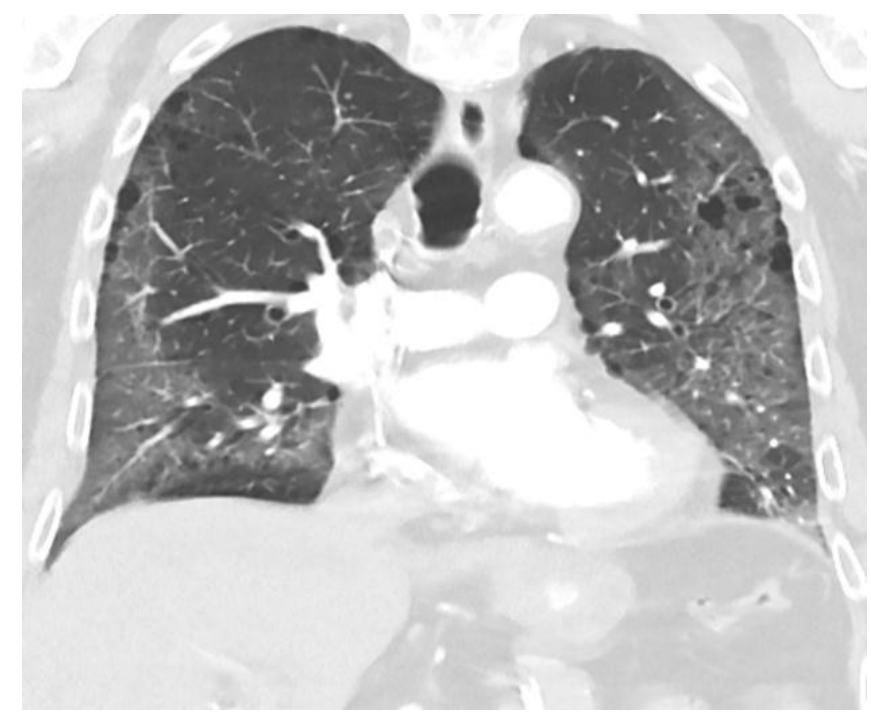

Figure 2: Both lungs CT image showing bilateral ground-glass opacities at the periphery in COVID 19 patient (Quoted from wikipedia.org) 
Why do some COVID19 patients with GGO not feel dyspnea? People feel dyspnea only if they have one of three: The first reason if there something obstructs the airway (not the case in COVID 19 cases). The second reason, when the carbon dioxide level increases in the blood, the breath increases, and the patient gasps to eliminate the excess $\mathrm{CO} 2$ exactly as when someone does exercise. The third one when there is pneumonia. Pneumonia is a lung disease; where the air sacs are filled with fluid or pus, causing a problem in the gas exchange. In a normal situation, if the patient has pneumonia, he complaints about chest pain, discomfort, shortness of breath as a result of the fast and hard work of the abdominal and chest muscles to force the lung to breathe. This breathe-hardness indicates the deficiency of lung compliance; it is the ease of lung movement during inhaling and exhaling, and due to the fluids-fill in the microscopic alveoli, the lungs become tauter and more inelastic. But when some patient's don't show any breathing symptoms although they have lung abnormalities such (GGO), the researchers suggest two prospective reasons for this phenomenon; the first one is attributed to the blood vessels-constriction. When some of the lung parts got injured, the blood vessels around those damaged parts constrict and push the blood to healthier places (rerouting) where the gas exchanges can take place. Therefore, by vasoconstriction, the patient body can sustain a relative level of the oxygen needed for life ${ }^{[17]}$.

The second reason is ascribed to the fluid buildup in the air sacs, in the initial phase of the disease, the fluid is not enough to stifle the lung or hinds gas exchanges. Therefore, the oxygen level is not as low to gasp ${ }^{[18]}$. The concern is how long does the lung survive in fluid existence?! GGO could be benign in asymptomatic patients and recover until the disease reaches its apogee. Ground glass opacities are well-known for hazy opacification as it appears on CT as "shades of grey" these grey areas indicate the lung illness because of the fluid which replaced the air in the air sacs ${ }^{[16,19]}$. These grey shades signify that not all the whole air sacs are filled with fluid. Therefore, the lung structure is not disordered. Although there are haziness areas on CT, the subsidiary structure of the lung can still be identified, such as the blood vessels, airways, and lung tissue. Indeed, there are some impairment areas in the lung but it still, works "it is similar to a glass that is still translucent but not shiny" Yale Medicine pulmonologist Jennifer Possick said. Therefore, it's possible to find COVID 19 patients don't feel shortness of breath as the oxygen level or Co2 don't be affected or gas exchange languished. In the contrast in the extremely diseased lung (severe pneumonia) appears on CT in white areas due to its full of puss or fluid ${ }^{[12]}$ (Figure 3).

Many of those mentioned cases weren't diagnosed initially, but they were identified by chance. For example, if someone has a complaint about gastrointestinal and had no about respiratory; when he/she was sent for abdominal CT, the radiologist found GGO as the abdominal CT catches the bottom of the lungs. Otherwise, this patient perhaps would develop silent lung damage and die ${ }^{[16]}$. Thus, the asymptomatic patient can be out of risk of silent lung damage by CT examination as it can early identify the COVID 19 pneumonia ${ }^{[20]}$.

The amazement is when some COVID19 patients develop pneumonia and the air sacs are almost filled with liquid, but they don't feel any breathing problems; or even that they have a significant low oxygen level (silent hypoxia). By the time passing, they felt, but after they became in critical condition; because they had severe pneumonia as seen in (Figure 3).

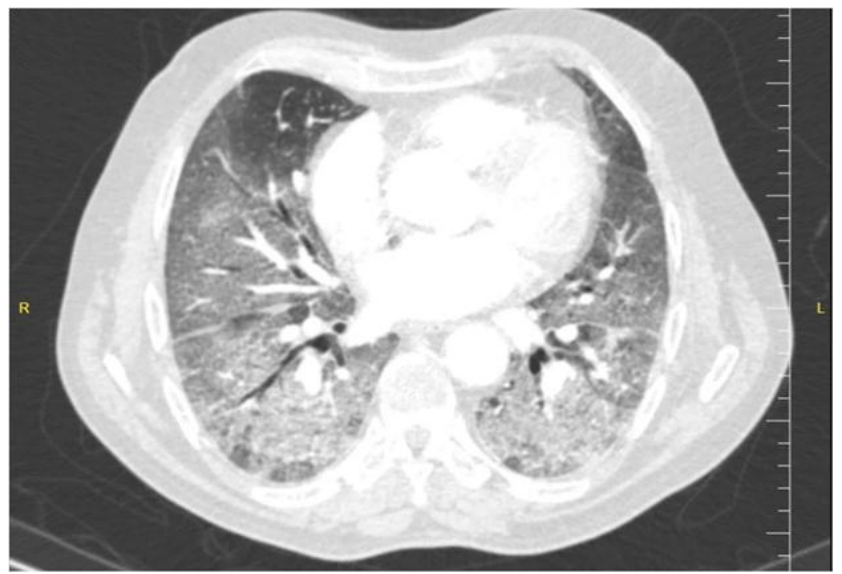

Figure 3: An axial CT image for a man chest with COVID-19 pneumonia shows extensive ground-glass opacities involving in all lower lobes in both lungs, giving a white appearance to the lung, with air bronchograms

(Quoted from Egyptian Journal of Radiology and Nuclear Medicine ${ }^{[21]}$ )

Silent hypoxia is unnoticeable oxygen lacking which can cause damage quietly, therefore called "silent", and because of its masked action, it's hard to recognize the illness severity and consequently delay medical care ${ }^{[22]}$. If the patient does not identify that he has hypoxia early as much as possible will not be able to avoid the deterioration or the silent damage to the vital organs, especially the lung.

The conundrum is that some COVID 19 patients who have severe hypoxia don't have corresponded symptoms to respiratory illness ${ }^{[23]}$. Recently, researchers have reported several cases of silent hypoxia. For instance, such a 72-year-old man, when arrived at the hospital, had no significant respiratory distress; but, he had extremely low oxygen saturation, and ultimately died due to respiratory failure with severe hypoxia ${ }^{[24]}$.

\section{Hypoxia mechanism}

At sea level, the ordinary oxygen saturation is 94 to $100 \%$, when COVID 19 patients have pneumonia, oxygen saturation reaches as low as 50 percent, which is incompatible with life. The researchers suggested an initial explanation of the hypoxia mechanism. They think when coronavirus strikes the patients' lungs attack the pneumocytes that make the surfactant. This surfactant preserves lung compliance by regulating alveolar surface tension to keep the air sacs open between breaths. When the virus attaches to Angiotensin-converting enzyme (ACE2) receptors then enters pneumocytes, causes downregulation of the lung enzymes, beside the detrimental inflammation effect of ACE2 leads to the air sacs collapsing. Thus the disabled parts cannot provide the bloodstream with oxygen causing oxygen levels to fall, which is called (hypoxia) ${ }^{[7,17,25]}$.

However, some studies suggest that hypoxia resulted from severe fluid buildup in the air sacs. On the other hand, some said that low oxygen saturation has no known reason and doesn't relate to pneumonia ${ }^{[18]}$. Also, some contradictory perspectives refer to that silent hypoxia could result from lung damage or cause silent lung damage. The question is, how does the hypoxic patient not show any related symptoms?! whether the hypoxia causes lung damage or resulted from lung damage. The proposed question of its importance is to find its answer to save those patients from sudden death which is the rational result of lung damage.

How COVID 19 patients with severe hypoxia still attentive and using their cellphones? 
There are many case reports about COVID 19 patients who had severe hypoxia, which it's supposed not compatible with life. Patients with severe hypoxia need intubation as they have low oxygen saturation as low as $50 \%$. With this lack of oxygen level, the patient should be unconscious or gasps to take his breath with terrible pain. However, the reported cases were alert and using their phones; they didn't show any signs of such acute hypoxia, and some of them had been discovered coincidentally ${ }^{[7,26]}$.

The bewildering of the silent hypoxia is the contradiction between good lung compliance and the disabled gas exchanges. The researchers to construe such baffling complications have provided some scenarios for how the patient has such oxygen deficiency without panting to take his breath ${ }^{[27]}$.

\section{The first scenario (Adaptation)}

When the body oxygen level begins to drop, the lung tries to compensate for this deficiency by breathing fast as much as it can (increases the breathing rate). Unfortunately, some patients don't aware of increasing their breathing rate, and thus they don't ask for help. Then, slowly the body becomes adapted to the new low level of oxygen. Although internally that patient about to have a crippled lung, the body doesn't feel that there is a problem to give an alarm. The reasearchers state that kind of adaptation resembles when someone goes to a higher altitude his body adapting to the new oxygen level ${ }^{[26]}$.

\section{The second scenario (Carbon expel)}

As a result of Coronavirus infection, the alveolar and interstitial inflammation disrupts gas exchange. Initially, the lung air sacs didn't heavy with fluid; therefore, they didn't stifle yet. Besides, as long as that substance (surfactant) within the normal range, prevents the alveoli from deflation, and keep the lung compliance. Due to the solubility and diffusion of $\mathrm{CO} 2$ are more than oxygen 20 times ${ }^{[23]}$, patients can expel carbon dioxide. Thus, there is no chance of excess $\mathrm{Co} 2$ (hypocapnic hypoxia no $\mathrm{Co} 2$ added) ${ }^{[28]}$, and without an increase of carbon dioxide level; patients do not feel breath shortness. Therefore, they weren't signaling breath problems or any discomfort ${ }^{[29]}$. Moreover, some empirical studies demonstrated that hypocapnic hypoxia is not always correlating to air hunger ${ }^{[30]}$

\section{The third scenario (Carotid body disorder)}

There are some suggestions that the virus is affecting the interoceptive sensory system and brain. This system senses the internal state of the body and sends signals to the brain. And by these neural pathways as (carotid body), the brain identifies the physiological state of the body, particularly the respiratory system $[31,32]$. The carotid sinus nerve is consolidated in the brain stem (Figure 4) to foment plenty of directed respiratory reflexes, mainly, to maintain the blood gases from changes ${ }^{[33]}$.

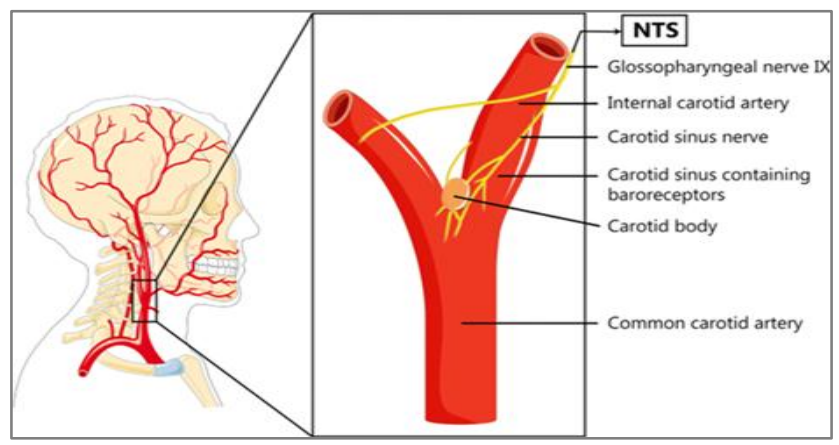

Figure 4: The carotid body location in the human body (Quoted from springer.com ${ }^{[34]}$ )
In the case of hypoxia, the sensor elements in the glomus cells of the carotid body detect the changes in the oxygen level, then release neurotransmitters such as Acetylcholine (ACh) which in turn stimulates the afferent fibers that send the signal to the central nervous system (CNS) as shown in (Figure 5). CNS responding to the signals by inducing reflex stimulation of the breathing and blood pressure ${ }^{[35]}$.

As several studies reported that ACE2 is considered a doorway for the SARS-COV-2 to enter the host cell, The researchers think when the virus binds to the ACE2 receptors on the carotid body cells, affects the ability of the glomus cells and its chemoreceptors, which is in charge of sensing the oxygen. Besides the prospective detrimental effect of ACE2 of Angiotensin II production, which can increase inflammation and cell death in the alveoli ${ }^{[36]}$. Therefore, the patient's body cannot recognize oxygen lacking, ${ }^{[29,37,38]}$ and thus, make the patient lose the mechanism of lack-oxygen sensing and will not have the opportunity to be warned.

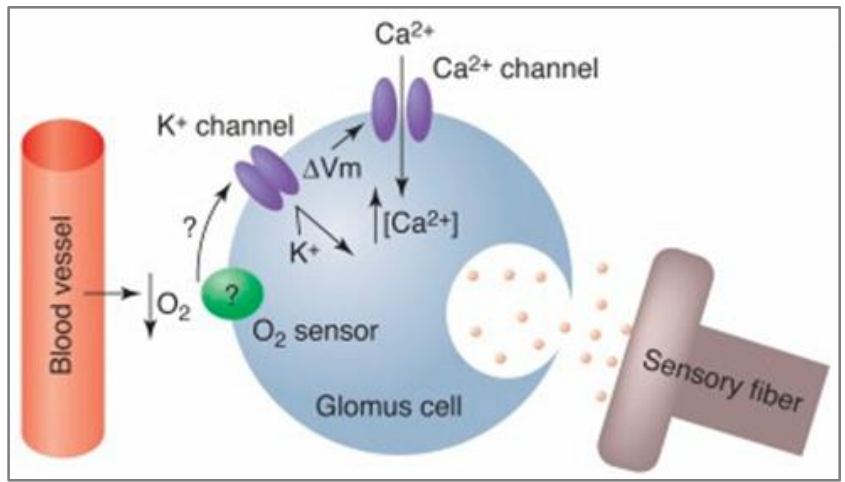

Figure 5: Membrane model of carotid body-glomus cells sensing oxygen.

(Quoted from Science Direct.com ${ }^{[39]}$ )

All the above information is an attempt to explain some ambiguities of COVID 19 situations and their reasons based on the recently published studies and case reports. However, this information is not a definite conclusion as the COVID 19 situation every day has new, but what certain is caution and take the following advice into account.

\section{Advice}

There are some recommendations and instructions that should be followed if the one suspected with COVID 19 to avoid the risk of silent complications. CT scans and Pulse oximeter are the crucial elements to eschew the insidious face of COVID19.

Studies found that CT scans can identify about $97 \%$ of COVID 19 cases even if they have no symptoms, whereas (RTPCR) can only identify about $30-50 \%$. , however, the blood test is only $59 \%$ of the real cases ${ }^{[12,20]}$.

The pulse oximeter can detect COVID 19 pneumonia by measuring blood oxygen saturation in the body; therefore, the early examination can identify the oxygen deficiency, which warns for breathing problems correlated to COVID pneumonia.

\section{Important note}

The aforementioned clinical examinations are for patients who whether confirmed positive Coronavirus or suspicious (high clinical suspicion of COVID-19, but testing negative) or even if the individual has only the common symptoms. The scrutiny detection by $\mathrm{CT}$ and follow-up by oximeter are extremely important as the 
earlier diagnosis, the more avoiding the ventilator or the sudden death.

\section{Conclusion}

Coronavirus perplexed whole physicians around the world, because of its vague expression as it differs from one to another. With the Coronavirus, if two persons have infected, you can find different ends for those two. One could end with mechanical ventilation; quite the contrast, the other wasn't even affected. Moreover, the virus ambiguity increased when the medical world found asymptomatic people, but developed silent damage and even ended with sudden death. There are many different suggested reasons for the absence of appropriate symptoms of lung abnormalities and oxygen deficiency in some patients. Thus, not necessarily if the individual has no symptoms, that he is out of risk. Therefore, the examination and follow-up by CT scan and pulse oximeter, giving an accurate result that protects them from the treatments which end with a mortality rate of about $80 \%{ }^{[40]}$.

\section{List of abbreviations}

Acute respiratory distress syndrome (ARDS)

World health organization (WHO)

Centers for Disease Control (CDC)

Ground-glass opacities (GGO)

Computed Tomography (CT)

Fluorescence reverse-transcriptase-polymerase chain reaction (FRT-PCR)

Angiotensin-converting enzyme (ACE)

Central nervous system (CNS)

Acetylcholine (ACh)

Reverse transcription-polymerase chain reaction (RT-PCR)

\section{References}

[1] Provided under "Latest Updates". Learn more about Worldometer's COVID-19 data.

[2] Novel Coronavirus (2019-nCoV) situation reports World Health Organization (WHO)

[3] COVID-19 Case Tracker. (2020) Johns Hopkins Coronavirus Resource Center.

[4] Wu Z and McGoogan JM (2019) Characteristics of and important lessons from the coronavirus disease (COVID19) outbreak in China: summary of a report of 72314 cases from the Chinese center for disease control and prevention. JAMA 2020.

[5] Shohei Inui , Akira Fujikawa, Motoyuki Jitsu, Naoaki Kunishima, Sadahiro Watanabe, et al. (2020) Chest CT Findings in Cases from the Cruise Ship Diamond Princess with Coronavirus Disease (COVID-19). J Radiology: Cardiothoracic Imaging Vol. 2, No. 2

[6] Tibi Puiu (2020) COVID-19 asymptomatic cases may still develop lung damage. J Health \& Medicine, News.

[7] Richard Levitan. (2020) The Infection That's Silently Killing Coronavirus Patients. New york times

[8] Vicky Pathare. (2020) Hypoxia big trigger of COVID-19 deaths; studies show many victims were absolutely asymptomatic until it was too late. J Pune Mirror.

[9] Mary Kekatos (2020) As many as half of coronavirus patients with NO symptoms may silently suffer 'disturbing' lung damage that leaves them oxygendeprived without knowing it. J mailonline
[10] John Kinnear. (2020) Coronavirus: asymptomatic people can still develop lung damage. J The conversation.

[11] M. Tsikala Vafea, E. Atalla, M. Kalligeros, E.K. Mylona, F. Shehadeh and E. Mylonakis (2020) Chest CT findings in asymptomatic cases with COVID-19: a systematic review and metaanalysis. Clinic.Radio. V75, issue 11.

[12] Leah Groth (2020) What Do 'Ground Glass Opacities' Mean in Lung Scans of COVID-19 Patients? Exp. Health.

[13] Herring W (2020) Learning radiology: recognizing the basics (4th ed.). Philadelphia: Elsevier. pp. 2

[14] Mettler Jr FA (2019). Essentials of radiology (Fourth ed.). Philadelphia, PA: Elsevier. pp. 299-331.

[15] Jump up to:a b c d Sharma A, Abbott G (2019). Thoracic imaging (Third ed.). Philadelphia, PA: Elsevier

[16] Kristina Fiore (2020) Hazy on Ground-Glass Opacities? Here's What They Are- Frequent finding in COVID-19, but how disease-specific is it? J Medpagetoday

[17] Boston University. (2020) Three reasons why COVID-19 can cause silent hypoxia: Biomedical engineers use computer modeling to investigate low blood oxygen in COVID-19. J patients. ScienceDaily.

[18] Stephanie Pappas (2020) 'Silent hypoxia' may be killing COVID-19 patients. But there's hope. J Live Science.

[19] Goodman LR (2015). Felson's principles of chest roentgenology (Fifth ed.). Philadelphia, PA: Elsevier. pp. Supplement 3, e36-e80.

[20] Heng Meng, a Rui Xiong, a Ruyuan He, a Weichen Lin, a Bo Hao, a Lin Zhang, et al. (2020) CT imaging and clinical course of asymptomatic cases with COVID-19 pneumonia at admission in Wuhan, China. J NCBI Infect. 81(1): e33-e39.

[21] Mohamed, Y.G., Mohamud, M.F.Y., Medişoğlu, M.S. et al. (2020) Clinical and chest CT presentations from 27 patients with COVID-19 pneumonia in Mogadishu, Somalia: a descriptive study. Egypt J Radiol Nucl Med 51,184

[22] Pulmonary Manifestations (2020) IDSA's Newsletter.

[23] Atanu Chandra, Uddalak Chakraborty, Jyotirmoy Pal and Parthasarathi Karmakar (2020) Silent hypoxia: a frequently overlooked clinical entity in patients with COVID-19. J BMJ Volume 13, Issue 9.

[24] Wilkerson RG, Adler JD, Shah NG, et al. (2020) Silent hypoxia: a harbinger of clinical deterioration in patients with COVID-19. Am J Emerg Med.

[25] Hoffmann M, Kleine-Weber H, Schroeder S, et al. (2020) SARS-CoV-2 cell entry depends on ACE2 and TMPRSS 2 and is blocked by a clinically proven protease inhibitor. Cell. 181:271-80.

[26] CNNWire (2020) Silent hypoxia: Coronavirus patients who should be gasping for air but aren't. J eyewit. News.

[27] Tobin MJ, Laghi F and Jubran A. (2020) Why COVID19 Silent Hypoxemia is Baffling to Physicians [published online. Am J Respir Crit Care Med.

[28] Harold J. Bell, Carrie Ferguson, Valerie Kehoe, and Philippe Haouzi (2009) Hypocapnia increases the prevalence of hypoxia-induced augmented breaths. J Ameri. Journal of physio

[29] Emily Henderson (2020) Silent hypoxia and its role in COVID-19 detection. J news med. Life scie.

[30] Ottestad Wand Søvik S (2020) COVID-19 patients with respiratory failure: what can we learn from aviation medicine? Br J Anaesth. 18:30226-9. 
[31] Craig, A. D. (2002) How do you feel? Interoception: the sense of the physiological condition of the body. J Nature Reviews Neuroscience. 3 (8): 655-666.

[32] G., Cameron and Oliver (2002) Visceral sensory neuroscience: interoception. J Oxford University Press.

[33] Maria Pedro Guarino and Sílvia Vilares Conde (2015) Caffeine, Insulin Resistance, and Hypertension. J scien.direc.

[34] Eline H. Groenland and Wilko Spiering (2020) Baroreflex Amplification and Carotid Body Modulation for the Treatment of Resistant Hypertension. J link.springer.V 27.

[35] Ying-Jie Peng and Nanduri R Prabhakar (2018) Measurement of Sensory Nerve Activity from the Carotid Body. J national liberary of med. Pub Med 1742:115-124.

[36] Amr Zaher and Sara Ali (2020) The Potential DoubleFaced Interactions of ACE Inhibitors/Blockers with
SARS-COV-2 inCardiovascular Diseases and Diabetes Mellitus Patients Who Developed COVID 19. Acta Scient. Pharma. Sci. V 4 Issue 8.

[37] Fung ML (2015) Expressions of angiotensin and cytokine receptors in the paracrine signaling of the carotid body in hypoxia and sleep apnea. J Respir Physiol Neurobiol. 209:6-12.

[38] Dhont S, Derom E, Van Braeckel E, Depuydt P and Lambrecht BN (2020) The pathophysiology of "happy" hypoxemia in COVID-19. J Respir Res. 28;21(1):198.

[39] JoséLópez-Barneo (2003) Oxygen and glucose sensing by carotid body glomus cells. J Scien.direc. 13 (4): 493 499.

[40] Jason Teo (2020) Early Detection of Silent Hypoxia in Covid-19 Pneumonia Using Smartphone Pulse Oximetry. J Med Syst. 44(8): 134 